BULLETIN OF THE

AMERICAN MATHEMATICAL SOCIETY

Volume 77, Number 6, November 1971

\title{
ON T-STRUCTURES
}

BY M. G. BARRATT

Communicated by Ed Brown, April 5, 1971

Let $\Gamma$ be the functor described in [1], such that $\Gamma X \equiv \Omega^{\infty} \Sigma^{\infty} X$, and let $\iota_{X}: X \rightarrow \Gamma X, h_{X}: \Gamma^{2} X \rightarrow \Gamma X$ be the natural transformations. A map $f: \Gamma X \rightarrow X$ will be called a $\Gamma$-structure on $X$ if

$$
f \circ \Gamma f=f \circ h_{X}, \quad f \circ \iota_{x}=\text { Identity. }
$$

Let $R$ be the functor from bisimplicial sets to simplicial sets such that

$$
(\Omega K)\left(\Delta^{n}\right)=K\left(\Delta^{n}, \Delta^{n}\right), \quad(R K)(\phi)=K(\phi, \phi)
$$

for all simplices $\Delta^{n}$ and all simplicial operators $\phi$. The realizations of $K$ and $\Omega K$ are homeomorphic.

Suppose $X$ has a given $\Gamma$-structure.

THEOREM. There is a functor $\mathbf{X}$ from simplicial sets to free bisimplicial groups such that

(i) $\pi_{*} \Omega \mathrm{QX}$ is a homology theory.

(ii) $X \equiv \Omega^{n} \cap X\left(S^{n}\right)$ for all $n \geqq 0$, and

$\mathrm{X}(A)=\left[\Gamma\left(A_{\wedge} X\right) \leftrightarrows \Gamma\left(A_{\wedge} \Gamma X\right) \leftrightarrows \Gamma\left(A_{\wedge} \Gamma^{2} X\right) \leftrightarrows \Gamma\left(A_{\wedge} \Gamma^{3} X\right) \leftrightarrows \cdots\right]$

Corollary. (i) If $(X, f)=\left(\Gamma Y, h_{Y}\right)$ then $\mathbb{a X} A \equiv \Gamma(A \wedge Y)$.

(ii) Let $\mathcal{E}=\left(a \times S^{n}\right)$ be the $\Omega$-spectrum then $\pi_{*}(a \times A) \cong H_{*}(A ; \mathcal{E})$.

It is easy to verify the exactness axiom, using the version of $\mathrm{G}$. W. Whitehead's Magic Exact Sequence given in [1], and a lemma that $R K$ is contractible if $K$ is a bigroup

$$
K=[K(0) \leftrightarrows K(1) \leftrightarrows K(2) \leftrightarrows \cdots]
$$

in which each level $K(n)$ is contractible. The method is sketched in [1].

There is an overlap with similar work by Anderson, by Beck, and by Quillen and Segal. Thanks are due to E. B. Curtis, P. J. Eccles, D. S. Kahn, D. M. Kan, M. E. Mahowald, S. Priddy and G. W. Whitehead.

\section{REFERENCES}

1. M. G. Barratt, A free group functor for stable homotopy, Proc. Sympos. Pure Math., vol. 22, Amer. Math. Soc., Providence, R. I., 1971, pp. 31-36.

Manchester University, Manchester, England

NORTHWESTERN UNIVERsity, Evanston, Illinois 60201

University of Washington, Seattle, Washington 98105

Copyright (c) American Mathematical Society 1971 